\title{
Fixed Points of Single- and Set-Valued Mappings in Uniformly Convex Metric Spaces with No Metric Convexity
}

\author{
Rafa Espínola, ${ }^{1}$ Aurora Fernández-León, ${ }^{1}$ and Bożena Piątek ${ }^{2}$ \\ ${ }^{1}$ Departamento de Análisis Matemático, Universidad de Sevilla, P.O. Box 1160, 41080 Sevilla, Spain \\ ${ }^{2}$ Institute of Mathematics, Silesian University of Technology, 44-100 Gliwice, Poland \\ Correspondence should be addressed to Rafa Espínola, espinola@us.es
}

Received 20 April 2009; Accepted 28 May 2009

Academic Editor: Mohamed A. Khamsi

Copyright (C) 2010 Rafa Espínola et al. This is an open access article distributed under the Creative Commons Attribution License, which permits unrestricted use, distribution, and reproduction in any medium, provided the original work is properly cited.

We study the existence of fixed points and convergence of iterates for asymptotic pointwise contractions in uniformly convex metric spaces. We also study the existence of fixed points for setvalued nonexpansive mappings in the same class of spaces. Our results do not assume convexity of the metric which makes a big difference when studying the existence of fixed points for set-valued mappings.

\section{Introduction}

This paper is motivated by the recent paper [1]. In [1] the authors study different questions related to fixed points of asymptotic pointwise contractive/nonexpansive mappings in CAT(0) spaces. CAT(0) spaces are studied in [1] as a very significant example within the class of uniformly convex metric spaces (the reader can consult [2] for details on CAT(0) spaces). In our present paper we propose to consider similar questions on uniformly convex metric spaces under the mildest additional conditions we may impose. More precisely, we will work with uniformly convex metric spaces with either a monotone modulus of convexity in the sense first given in [3] or a lower semicontinuous from the right modulus of convexity (see Section 2 for proper definitions). For a recent survey on the existence of fixed points in geodesic spaces, the reader may check [4], for recent achievements on related topics the reader may also check [5].

The notion of asymptotic pointwise contractions was introduced in [6]. Then it was also studied in [7] where, by means of ultrapower techniques, different results about the 
existence of fixed points and convergence of iterates were proved. In [8] new proofs were presented but this time after applying only elementary techniques. Very recently, in [1], these techniques were applied in CAT(0), where the authors attend to the Bruhat-Tits inequality for CAT(0) spaces in order to obtain such results. In the present paper we show that actually most of those results still hold for general uniformly convex metric spaces under mild conditions on the modulus of convexity. In Section 3 we focus on single-valued mappings and, in particular, on mappings which are asymptotically pointwise contractive/nonexpansive to study the existence of fixed points, convergence of Picard's iterates, and the structure of their sets of fixed points. As a technical result we need to show that bounded sequences in these spaces have a unique asymptotic center which, as a by-product, leads to Kirk's Fixed Point Theorem. In Section 4 we study different problems regarding set-valued mappings in these spaces. The main technical difficulty to achieve similar results to those shown in [1] is that now we cannot count on the existence of fixed points for nonexpansive setvalued mappings for the kind of spaces we deal with. Finding fixed point for set-valued nonexpansive mappings in uniformly convex metric spaces was first studied by Shimizu and Takahashi [9], where the existence of fixed points was guaranteed under stronger conditions on the modulus of convexity and the additional condition of metric convexity of the space. The fact that we do not have that the metric are convex will make the problem more complicated and this will take us to impose new conditions on the modulus of convexity which we will relate with the geometry of the space.

\section{Basic Definitions and Results}

We introduce next some basic definitions.

Definition 2.1. Let $(X, d)$ be a metric space. A mapping $T: X \rightarrow X$ is called a pointwise contraction if there exists a mapping $\alpha: X \rightarrow[0,1)$ such that

$$
d(T(x), T(y)) \leq \alpha(x) d(x, y)
$$

for any $y \in X$.

It is proved in [8] (see also [6]) that if $K$ is a weakly compact convex subset of a Banach space and $T: K \rightarrow K$ is a pointwise contraction, then $T$ has a unique fixed point and the sequence of the iterates of $T$ converges to the fixed point for any $x \in K$. As it is pointed out in [1], the uniqueness of fixed points and convergence of iterates for these mappings directly follow if existence is guaranteed.

Definition 2.2. Let $(X, d)$ be a metric space. Let $T: X \rightarrow X$ be a mapping, and let $\alpha_{n}: X \rightarrow$ $[0, \infty)$ for each $n \in \mathbb{N}$ be such that

$$
d\left(T^{n}(x), T^{n}(y)\right) \leq \alpha_{n}(x) d(x, y) \text { for any } y \in X
$$

Then

(i) $T$ is called an asymptotic pointwise contraction if $\left\{\alpha_{n}\right\}$ converges pointwise to $\alpha$ : $X \rightarrow[0,1)$; 
(ii) $T$ is called an asymptotic pointwise nonexpansive mapping if $\lim \sup \alpha_{n}(x) \leq 1$ for any $x \in X$;

(iii) $T$ is called a strongly asymptotic pointwise contraction if $\lim \sup \alpha_{n}(x) \leq k$, with $0<k<1$, for any $x \in X$.

In this paper we will mainly work with uniformly convex geodesic metric space. Since the definition of uniform convexity requires the existence of midpoints, the word geodesic is redundant and so, for simplicity, we will usually omit it.

Definition 2.3. A geodesic metric space $(X, d)$ is said to be uniformly convex if for any $r>0$ and any $\varepsilon \in(0,2]$ there exists $\delta \in(0,1]$ such that for all $a, x, y \in X$ with $d(x, a) \leq r, d(y, a) \leq r$ and $d(x, y) \geq \varepsilon r$ it is the case that

$$
d(m, a) \leq(1-\delta) r
$$

where $m$ stands for any midpoint of any geodesic segment $[x, y]$. A mapping $\delta:(0,+\infty) \times$ $(0,2] \rightarrow(0,1]$ providing such a $\delta=\delta(r, \varepsilon)$ for a given $r>0$ and $\varepsilon \in(0,2]$ is called a modulus of uniform convexity.

Notice that this definition of uniform convex metric spaces is weaker than the one used in [9] in two ways. First, we do not impose that the metric is convex and, second, our modulus of convexity does depend on the two variables $r$ and $\varepsilon$ while it is assumed to depend only on $\varepsilon$ in [9].

Definition 2.4. Let $(X, d)$ be a metric space, then the metric is said to be convex if for any $x, y$ and $z$ in $X$, and $m$ a midpoint in between $x$ and $y$,

$$
d(z, m) \leq 1 / 2(d(z, x)+d(z, y)) .
$$

It is easy to see that uniformly convex metric spaces are uniquely geodesic, that is, for each two points there is just one geodesic joining them. Therefore midpoints and geodesic segments $[x, y]$ joining two points are unique. In this case there is a natural way to define convexity. A subset $C$ of a (uniquely) geodesic space is said to be convex if $[x, y] \subseteq C$ for any $x, y \in C$. For more about geodesic spaces the reader may check [2].

To obtain our results we will need to impose additional conditions on the modulus of convexity. Following $[3,10]$ we consider the notion of monotone modulus of convexity as follows.

Definition 2.5. If a uniformly convex metric space $X$ admits a modulus of convexity $\delta$ such that it decreases with $r$ (for each fixed $\varepsilon$ ) then we say that $\delta$ is a monotone modulus of convexity for $X$.

In the same way we define a lower semicontinuous from the right modulus of convexity as follows.

Definition 2.6. If a uniformly convex metric space $X$ admits a modulus of convexity $\delta$ such that it is lower semicontinuous from the right with respect to $r$ (for each fixed $\varepsilon$ ) then we say $\delta$ is a lower semicontinuous from the right modulus of convexity for $X$. 
Let $X$ be a metric space and $\mp$ a family of subsets of $X$. Then, following [1], we say that $F$ defines a convexity structure on $X$ if it contains the closed balls and is stable by intersection.

Let $X$ be a metric space and $\mp$ a convexity structure on $X$. Given $\Phi: X \rightarrow[0, \infty)$, we say that $\Phi$ is $\mathcal{F}$-convex if $\{x: \Phi(x) \leq r\} \in \mathcal{F}$ for any $r \geq 0$.

If we consider a bounded sequence $\left\{x_{n}\right\}$ in $X$, we are able to define a function $r\left(\cdot, x_{n}\right)$, called type, such that for each $x$

$$
r\left(x, x_{n}\right)=\limsup _{n \rightarrow \infty} d\left(x_{n}, x\right) .
$$

The asymptotic center of a bounded sequence with respect to a subset $C$ of $X$ is then defined as

$$
A_{C}\left(\left\{x_{n}\right\}\right)=\left\{x \in X: r\left(x, x_{n}\right) \leq r\left(y, x_{n}\right) \text { for any } y \in C\right\} \text {. }
$$

If the asymptotic center is taken with respect to $X$ then it is simply denoted by $A\left(\left\{x_{n}\right\}\right)$.

Definition 2.7. We say that a convexity structure is $T$-stable if types are $\mathcal{F}$-convex.

In [1] the following definition of compactness for convexity structure was considered.

Definition 2.8. Given $\mathcal{F}$ a convexity structure, we will say that $\mathcal{F}$ is compact if any family $\left(A_{\alpha}\right)_{\alpha \in \Gamma}$ of elements of $\mathcal{F}$ has nonempty intersection provided $\cap_{\alpha \in F} \neq \emptyset$ for any finite subset $F \subset \Gamma$.

In our paper we will rather use the idea of compactness given in [11]. Notice that this second notion of compactness is weaker than the previous one.

Definition 2.9. Given $\mathcal{F}$ a convexity structure, we will say that $\mathcal{F}$ is nested compact if any decreasing chain $\left(A_{\alpha}\right)_{\alpha \in \Gamma}$ of nonempty bounded elements of $\mathcal{F}$ has nonempty intersection.

A very important property given in [3] about complete uniformly convex metric spaces with monotone modulus of convexity is that decreasing sequences of nonempty bounded closed and convex subsets of these spaces have nonempty intersection. As a consequence, we have that if $\mathcal{F}$ stands for the collection of nonempty closed and convex subsets of a complete uniformly convex metric space with monotone modulus of convexity, then $\mathcal{F}$ is a nested compact convexity structure.

Remark 2.10. It is not hard to see that the same remains true if the monotone condition on the modulus is replaced by lower semicontinuity from the right.

\section{Asymptotic Pointwise Contractions in Uniformly Convex Metric Spaces}

In this section we give different results for the above defined mappings in uniformly convex metric spaces. Although, for expository reasons, our results will be usually proved only for uniformly convex metric spaces with a monotone modulus of convexity, they also hold when there is a lower semicontinuous modulus of convexity. Some indications about differences in both cases will be given. We begin with a technical result. 
Proposition 3.1. Let $(X, d)$ be a complete uniformly convex metric space with a monotone (or lower semicontinuous from the right) modulus of convexity $\delta(r, \varepsilon)$. Consider the family $\mathcal{F}$ of all nonempty closed and convex subsets of $X$. Then $F$ defines a nested compact and T-stable convexity structure on $X$.

Proof. It only remains to be proved that $\mathcal{F}$ is $T$-stable. Let $\left\{x_{n}\right\}$ be a bounded sequence in $X$ and consider the type defined by $\left\{x_{n}\right\}$. We need to show that $C_{r}=\left\{x: r\left(x, x_{n}\right) \leq r\right\} \in \mathcal{F}$ for any positive $r$. It is immediate to see that $C_{r}$ is closed and nonempty. To see that $C_{r} \in \mathcal{F}$ is also convex, consider $x$ and $y$ to be two different points in $C_{r}$. There is no restriction if we assume that $\lim \sup d\left(y, x_{n}\right) \leq \limsup d\left(x, x_{n}\right)=r_{1} \leq r$. Let $m$ be the midpoint of the segment $[x, y]$ and take $\varepsilon_{1}=d(x, y) /(r+1)$, then, by uniform convexity, we have that

$$
\begin{aligned}
d\left(m, x_{n}\right) & \leq\left(1-\delta\left(\max \left\{d\left(x, x_{n}\right), d\left(y, x_{n}\right)\right\}, \varepsilon_{1}\right)\right) \max \left\{d\left(x, x_{n}\right), d\left(y, x_{n}\right)\right\} \\
& <\max \left\{d\left(x, x_{n}\right), d\left(y, x_{n}\right)\right\}
\end{aligned}
$$

and so,

$$
\limsup d\left(m, x_{n}\right) \leq \lim \sup \max \left\{d\left(x, x_{n}\right), d\left(y, x_{n}\right)\right\}=r_{1} \leq r
$$

Hence, $m \in C_{r}$.

The following theorems were proved in [1] under the hypothesis of compactness on the convexity structure. We state it, however, under the hypothesis of nested compactness since this is all it is actually required in the proofs given in [1].

Theorem 3.2. Let $X$ be a bounded metric space. Assume that the convexity structure $A(M)$ is nested compact. Let $T: X \rightarrow X$ be a pointwise contraction. Then $T$ has a unique fixed point $x_{0}$. Moreover the orbit $\left\{T^{n}(x)\right\}$ converges to $x_{0}$, for each $x \in X$.

Theorem 3.3. Let $X$ be a bounded metric space. Assume that the convexity structure $A(M)$ is nested compact. Let $T: X \rightarrow X$ be a strongly asymptotic pointwise contraction. Then $T$ has a unique fixed point $x_{0}$. Moreover the orbit $\left\{T^{n}(x)\right\}$ converges to $x_{0}$, for each $x \in X$.

Now the next corollary follows.

Corollary 3.4. The above theorems hold for complete bounded uniformly convex metric spaces with either monotone or lower semicontinuous from the right modulus of convexity.

The following lemma is immediate.

Lemma 3.5. Let $X$ be a metric space and $F$ a nested compact convexity structure on $X$ which is $T$-stable. Then for any type $r\left(\cdot, x_{n}\right)$, there exists $x_{0} \in X$ such that

$$
r\left(x_{0}, x_{n}\right)=\inf \left\{r\left(x, x_{n}\right): x \in X\right\} .
$$

As a direct consequence of Proposition 3.1 and the previous lemma we get the following result for asymptotic pointwise contractions. We omit the details of its proof as it follows similar patterns as in [1, Theorem 4.2]. 
Theorem 3.6. Let $(X, d)$ be a complete uniformly convex metric space with a monotone (or lower semicontinuous from the right) modulus of convexity $\delta(r, \varepsilon)$. Suppose $X$ is bounded. Then every $T$ : $X \rightarrow X$ asymptotic pointwise contraction has a unique fixed point $x_{0}$. Moreover, the orbit $\left\{T^{n}(x)\right\}$ converges to $x_{0}$ for each $x \in X$.

Next we show some consequences of Proposition 3.1 and Lemma 3.5. The cases for monotone and lower semicontinuous from the right modulus of convexity are shown separately as they require different proofs.

Corollary 3.7. Let $X$ be a complete uniformly convex metric space with a monotone modulus of convexity and $\left\{x_{n}\right\}$ a bounded sequence in $X$. Then the set of asymptotic centers of $\left\{x_{n}\right\}$ is a singleton.

Proof. Let $u$ and $v$ be two different points in $A\left(\left\{x_{n}\right\}\right)$, and let $m$ be the midpoint of $[u, v]$. Let $r=r\left(u, x_{n}\right)=r\left(u, x_{n}\right), c=r+1$, and $\varepsilon_{1}=d(u, v) / c$. By the uniform convexity, there exists $N \in \mathbb{N}$ such that for every $n \geq N$,

$$
\begin{aligned}
d\left(m, x_{n}\right) & \leq\left(1-\delta\left(\max \left\{d\left(u, x_{n}\right), d\left(v, x_{n}\right)\right\}, \varepsilon_{1}\right)\right) \max \left\{d\left(u, x_{n}\right), d\left(v, x_{n}\right)\right\} \\
& \leq\left(1-\delta\left(c, \varepsilon_{1}\right)\right) \max \left\{d\left(u, x_{n}\right), d\left(v, x_{n}\right)\right\} .
\end{aligned}
$$

If we let $n$ go to infinite, we obtain that $r\left(m, x_{n}\right) \leq\left(1-\delta\left(c, \varepsilon_{1}\right)\right) r<r$, which is clearly a contradiction.

Remark 3.8. This corollary has been first proved in [12, Proposition 3.3] for a certain class of uniformly convex hyperbolic spaces with monotone modulus of convexity.

Now we show the lower semicontinuous case.

Corollary 3.9. Let $X$ be a complete uniformly convex metric space with a lower semicontinuous from the right modulus of convexity and $\left\{x_{n}\right\}$ a bounded sequence in $X$. Then the set of asymptotic centers of $\left\{x_{n}\right\}$ is a singleton.

Proof. Let $u$ and $v$ be two different points in $A\left(\left\{x_{n}\right\}\right)$ and let $m$ be the midpoint of $[u, v]$. Let $r=r\left(u, x_{n}\right)=r\left(v, x_{n}\right), \varepsilon=d(u, v) /(r+1)$, and let us fix $p \in \mathbb{N}$. Then $\max \left\{d\left(u, x_{n}\right), d\left(v, x_{n}\right)\right\} \leq$ $r+p^{-1}$ for each $n$ large enough. By the uniform convexity,

$$
d\left(m, x_{n}\right) \leq\left(1-\delta\left(r+p^{-1}, \varepsilon\right)\right)\left(r+p^{-1}\right)
$$

for the same $n$ as above and finally

$$
r\left(m, x_{n}\right) \leq\left(1-\delta\left(r+p^{-1}, \varepsilon\right)\right)\left(r+p^{-1}\right)
$$

Now it suffices to observe that

$$
\begin{gathered}
\delta\left(r+p^{-1}, \varepsilon\right) \geq \frac{1}{2} \delta(r, \varepsilon) \\
1-\delta\left(r+p^{-1}, \varepsilon\right) \leq 1-\frac{1}{2} \delta(r, \varepsilon)
\end{gathered}
$$


for $p$ large enough. Combining it with (3.6) and taking $\lim _{p \rightarrow \infty}$ we obtain $r\left(m, x_{n}\right)<r$ as in the former corollary, and thus the contradiction.

Another consequence is Kirk Fixed Point Theorem in uniformly convex metric spaces.

Corollary 3.10. Let $X$ be a complete uniformly convex geodesic metric space with a monotone (or lower semicontinuous from the right) modulus of convexity. Suppose $\mathrm{X}$ is bounded, then any nonexpansive mapping $T: X \rightarrow X$ has a fixed point.

Proof. Consider $x \in X$ and $\left\{T^{n}(x)\right\}$ the sequence of its iterates. Let $\omega$ be the only asymptotic center of $\left\{T^{n}(x)\right\}$ in $X$. Then, by the nonexpansiveness of $T$, it follows that $r\left(T(\omega), T^{n}(x)\right) \leq$ $r\left(\omega, T^{n}(x)\right)$ and so, $T(\omega)=\omega$.

Now we present a counterpart for [1, Theorem 5.1].

Theorem 3.11. Let $(X, d)$ be a complete uniformly convex metric space with a monotone (or lower semicontinuous from the right) modulus of convexity $\delta(r, \varepsilon)$. Let $C$ be a bounded closed convex nonempty subset of $X$. Then any $T: C \rightarrow C$ asymptotic pointwise nonexpansive mapping has a fixed point, and the set of fixed points of $T, \operatorname{Fix}(T)$, is closed and convex.

Proof. Let $x \in C$ and consider $x_{n}=T^{n}(x)$. From Corollary 3.7, we know that $A_{C}\left(\left\{x_{n}\right\}\right)$ is a singleton. Let $\omega$ be the only point in that set, that is, $\omega$ is such that $r\left(\omega, x_{n}\right)=\inf \left\{r\left(u, x_{n}\right): u \in\right.$ $C\}$. We want to show that $\left\{T^{m}(\omega)\right\}$ is a Cauchy sequence. Suppose this is not the case. Then there exists a separated subsequence $\left\{T^{m_{i}}(\omega)\right\}$ of $\left\{T^{m}(\omega)\right\}$, that is, there exists $\varepsilon>0$ such that $d\left(T^{m_{k}}(\omega), T^{m_{h}}(\omega)\right) \geq \varepsilon$ for every $k \neq h$ in $\mathbb{N}$.

Let $m_{k h}$ be the midpoint of the segment $\left[T^{m_{k}}(\omega), T^{m_{h}}(\omega)\right], c=\operatorname{diam}(C)$ and $\varepsilon_{1}=\varepsilon / c$. The uniform convexity of the space, together with its monotone character, implies that for every $k$ and $h$ in $\mathbb{N}$

$$
\begin{aligned}
d\left(m_{k h}, x_{n}\right) & \leq\left(1-\delta\left(\max \left\{d\left(T^{m_{h}}(\omega), x_{n}\right), d\left(T^{m_{k}}(\omega), x_{n}\right)\right\}, \varepsilon_{1}\right)\right) \max \left\{d\left(T^{m_{h}}(\omega), x_{n}\right), d\left(T^{m_{k}}(\omega), x_{n}\right)\right\} \\
& \leq\left(1-\delta\left(c, \varepsilon_{1}\right)\right) \max \left\{d\left(T^{m_{h}}(\omega), x_{n}\right), d\left(T^{m_{k}}(\omega), x_{n}\right)\right\} .
\end{aligned}
$$

Notice that, by definition of $T$,

$$
r\left(T^{m}(\omega), x_{n}\right) \leq \alpha_{m}(\omega) r\left(\omega, x_{n}\right) .
$$

Then, if we let $n$ go to infinity,

$$
\begin{aligned}
r\left(\omega, x_{n}\right) & \leq r\left(m_{k h}, x_{n}\right) \\
& \leq\left(1-\delta\left(c, \varepsilon_{1}\right)\right) \max \left\{r\left(T^{m_{k}}(\omega), x_{n}\right), r\left(T^{m_{h}}(\omega), x_{n}\right)\right\} \\
& \leq\left(1-\delta\left(c, \varepsilon_{1}\right)\right) \max \left\{\alpha_{m_{k}}(\omega) r\left(\omega, x_{n}\right), \alpha_{m_{h}}(\omega) r\left(\omega, x_{n}\right)\right\}
\end{aligned}
$$

Since $T$ is pointwise asymptotic nonexpansive, then

$$
r\left(\omega, x_{n}\right) \leq\left(1-\delta\left(c, \varepsilon_{1}\right)\right) r\left(\omega, x_{n}\right),
$$


and so $r\left(\omega, x_{n}\right)=0$, which is a contradiction since, in virtue of (3.9), this implies that $T^{m}(\omega)$ converges to $\omega$. Therefore, $\left\{T^{m}(\omega)\right\}$ is a Cauchy sequence and its limit, again by (3.9), is $\omega$. Then, from the continuity of $T, T(\omega)=\omega$.

In consequence, $\operatorname{Fix}(T)$ is nonempty. Now, since $T$ is continuous, $\operatorname{Fix}(T)$ is closed. We show next that $\operatorname{Fix}(T)$ is also convex. Let $u, v$ be two different points in $\operatorname{Fix}(T)$ and $w$ the midpoint of the segment $[u, v]$. We need to show that $w \in \operatorname{Fix}(T)$. Now, since $T$ is pointwise asymptotic nonexpansive,

$$
d\left(u, T^{n}(w)\right)=d\left(T^{n}(u), T^{n}(w)\right) \leq \alpha_{n}(w) d(u, w)=\frac{\alpha_{n}(w) d(u, v)}{2}
$$

and, equally,

$$
d\left(v, T^{n}(w)\right)=d\left(T^{n}(v), T^{n}(w)\right) \leq \alpha_{n}(w) d(v, w)=\frac{\alpha_{n}(w) d(u, v)}{2} .
$$

Therefore, for $\varepsilon>0$, there exists $n_{0}$ such that if $n \geq n_{0}$ then

$$
T^{n}(w) \in B\left(u, \frac{d(u, v)}{2}+\varepsilon\right) \cap B\left(v, \frac{d(u, v)}{2}+\varepsilon\right)=D_{\varepsilon}
$$

but, from the proof of Proposition 2.2 in [3], the diameters of the sets $D_{\varepsilon}$ tend to 0 as $\varepsilon$ tends to 0 and so $\lim T^{n}(w)=w$, which proves $w$ is a fixed point of $T$.

Remark 3.12. The proof for the lower semicontinuous case follows in a similar way but following the reasoning of Corollary 3.9.

In [1] a demiclosed principle is also given for asymptotic pointwise nonexpansive mappings in CAT(0) spaces. Next we show that an equivalent result is also possible for uniformly convex metric spaces. Following [1] we define

$$
\left\{x_{n}\right\} \rightarrow_{C} \omega \text { if and only if } r\left(\omega, x_{n}\right)=\inf _{x \in C} r\left(x, x_{n}\right),
$$

where $C$ is a closed and convex subset of a uniformly convex metric space containing the bounded sequence $\left\{x_{n}\right\}$. Notice that this definition does not depend on the set $C$ when the space $X$ is a complete CAT(0) space. This is due to the fact that the asymptotic center of a bounded sequence of a complete CAT( 0 ) space belongs to the closed convex hull of the sequence, which easily follows from the very well-known fact that the metric projection onto closed convex subsets of a complete CAT(0) space is nonexpansive (see [2] for details). Recall that the existence and uniqueness of such a $\omega \in C$ in a complete uniformly convex metric spaces with monotone modulus of convexity is guaranteed by Corollary 3.7.

Proposition 3.13. Let $(X, d)$ be a complete uniformly convex metric space with a monotone modulus of convexity $\delta(r, \varepsilon)$. Let $C$ be a bounded closed convex nonempty subset of $X$. Let $T: C \rightarrow C$ an asymptotic pointwise nonexpansive mapping. Let $\left\{x_{n}\right\} \in C$ be an approximate fixed point sequence, that is, $\lim _{n \rightarrow \infty} d\left(x_{n}, T\left(x_{n}\right)\right)=0$, and such that $x_{n} \rightarrow \omega$ for a certain $\omega \in C$. Then $T(\omega)=\omega$. 
Proof. Since $\left\{x_{n}\right\}$ is an approximate fixed point sequence, then we have that

$$
r\left(x, x_{n}\right)=\limsup _{n \rightarrow \infty} d\left(x, T^{m}\left(x_{n}\right)\right)=r\left(x, T^{m}\left(x_{n}\right)\right)
$$

for any $m \geq 1$ ( see Note Added in Proof at the end of the paper). In consequence, since $r\left(T^{m}(x), T^{m}\left(x_{n}\right)\right) \leq \alpha_{m}(x) r\left(x, x_{n}\right)$ for $x \in C,(3.9)$ holds for any $x$.

Therefore, particularizing for $\omega$, we have that $\lim \sup _{m \rightarrow \infty} r\left(T^{m}(\omega), x_{n}\right)=r\left(\omega, x_{n}\right)$. Now we claim that $T^{m}(\omega) \rightarrow \omega$ as $m \rightarrow \infty$. Suppose on the contrary that there exist an $\varepsilon>0$ and a subsequence $\left\{T^{m_{k}}(\omega)\right\}$ of $\left\{T^{m}(\omega)\right\}$ such that $d\left(T^{m_{k}}(\omega), \omega\right) \geq \varepsilon$ for every $k \in \mathbb{N}$. Let $\omega_{m_{k}}$ be the midpoint of the geodesic segment $\left[T^{m_{k}}(\omega), \omega\right], c=\operatorname{diam}(C)$ and $\varepsilon_{1}=\varepsilon / c$. By uniform convexity, for every $k$, we have that

$$
\begin{aligned}
d\left(\omega_{m_{k}}, x_{n}\right) & \leq\left(1-\delta\left(\max \left\{d\left(\omega, x_{n}\right), d\left(T^{m_{k}}(\omega), x_{n}\right)\right\}, \varepsilon_{1}\right)\right) \max \left\{d\left(\omega, x_{n}\right), d\left(T^{m_{k}}(\omega), x_{n}\right)\right\} \\
& \leq\left(1-\delta\left(c, \varepsilon_{1}\right)\right) \max \left\{d\left(\omega, x_{n}\right), d\left(T^{m_{k}}(\omega), x_{n}\right)\right\} .
\end{aligned}
$$

If we consider the upper limit of the above inequality when $n \rightarrow \infty$, we get

$$
r\left(\omega, x_{n}\right) \leq r\left(\omega_{m_{k}}, x_{n}\right) \leq\left(1-\delta\left(c, \varepsilon_{1}\right)\right) \max \left\{r\left(\omega, x_{n}\right), r\left(T^{m_{k}}(\omega), x_{n}\right)\right\} .
$$

If we do the same when $k \rightarrow \infty$, we finally obtain that $r\left(\omega, x_{n}\right) \leq\left(1-\delta\left(c, \varepsilon_{1}\right)\right) r\left(\omega, x_{n}\right)$. Therefore $r\left(\omega, x_{n}\right)=0$, and the existence of fixed point follows the same as in Theorem 3.11.

Remark 3.14. The proof for the lower semicontinuous case follows in a similar way but following the reasoning of Corollary 3.9.

\section{Fixed Points of Set-Valued Mappings}

In this section we present fixed points theorems for set-valued mappings defined on uniformly convex metric spaces. Results stated for uniformly convex metric space with a monotone modulus of convexity also hold if there is a lower semicontinuous from the right modulus of convexity. Proofs of this second case will be omitted as they are based on technical results already proved for both kinds of modulus in Section 3. The Hausdorff metric on the closed and bounded parts of a metric space $X$ is defined as follows. If $U$ and $V$ are bounded and closed subsets of a metric space $X$, then

$$
H(U, V)=\inf \left\{\varepsilon>0: U \subseteq N_{\varepsilon}(V), V \subseteq N_{\varepsilon}(U)\right\},
$$

where $N_{\varepsilon}(V)=\{y \in X: \operatorname{dist}(y, V)=\inf \{d(y, x): x \in V\}<\varepsilon\}$. Let $C$ be a subset of a metric space $X$. A mapping $T: C \rightarrow 2^{X}$ with nonempty bounded closed values is nonexpansive if

$$
H(T(x), T(y)) \leq d(x, y)
$$

for all $x, y \in C$. Our main goal in this section is to study if given $X$ is a bounded uniformly convex metric space with monotone modulus of convexity, then every nonexpansive 
mapping $T: X \rightarrow 2^{X}$ with nonempty and compact values has a fixed point, that is, a point $x \in X$ such that $x \in T(x)$. This problem was first solved in the affirmative by Shimizu and Takahashi in [9] under the assumption that the metric is convex. If we lack this condition the problem is much more complicated and we can offer only partial answers. Our first answer will be achieved after imposing condition (i):

(i) there exists a point $x \in X$ such that for each $t \in(0,1)$ there is a number $s(t) \in(0,1)$ such that for all $y, z \in X$ :

$$
d(u, v) \leq s(t) d(y, z),
$$

where $u, v$ stand for points from geodesic segments $[x, y]$ and $[x, z]$, respectively, and $d(x, u)=t d(x, y), d(x, v)=t d(x, z)$.

Remark 4.1. Condition (i) can be seen as a kind of very weak hyperbolicity condition. In fact, it is immediate to see that hyperbolic uniformly convex spaces studied in $[3,10,12]$ satisfy condition (i) as well as for any uniformly convex $\mathrm{CAT}(k)$ space with $k \in \mathbb{R}$. Notice here that $\mathrm{CAT}(k)$ spaces with $k \leq 0$ are particular examples of hyperbolic uniformly convex spaces.

Theorem 4.2. Let $(X, d)$ be a complete uniformly convex metric space with a monotone modulus of convexity. Suppose that $X$ is bounded and the condition (i) holds true. Then each nonexpansive set-valued mapping $T: X \rightarrow 2^{X} \backslash\{\emptyset\}$ with compact values has a fixed point.

Proof. Let us fix $x_{0} \in X$ satisfying the condition (i). Now, from (i) and the fact that $T(x)$ is compact for any $x$, it follows that set-valued mappings $T_{t}: X \rightarrow 2^{X}, t \in(0,1)$ defined by

$$
T_{t}(x)=\left\{u \in X: \exists y \in T(x) \text { s.t. } u \in\left[x_{0}, y\right], d\left(x_{0}, u\right)=t d\left(x_{0}, y\right)\right\}
$$

are compact-valued contractions with constants $s(t)$. Nadler's Fixed Point Theorem for setvalued contractions implies that $T_{t}$ has a fixed point for each $t$ and therefore $T$ has an approximate fixed point sequence, that is, a sequence $\left\{x_{n}\right\}$ such that $\lim \operatorname{dist}\left(x_{n}, T\left(x_{n}\right)\right)=0$. According to Corollary 3.7 there is a unique asymptotic center of $\left\{x_{n}\right\}$ in X. Now the rest of the proof follows the same patterns of the standard one for uniformly convex Banach spaces (see [13, Theorem 15.3, page 165]).

It is wellknown that a CAT(1) space needs not to be uniformly convex if its diameter is not smaller than $\pi / 2$. Next we show, however, that the same above idea can be applied to CAT(1) spaces of radius smaller than $\pi / 2$. Remember that for $k>0$ the number $D_{k}$ is defined as $D_{k}=\pi / \sqrt{k}$ and that the radius of a bounded subset $C$ of $X$ is given by

$$
\operatorname{rad}_{X}(C)=\inf \{r: \text { there exists } x \in \mathrm{X} \text { such that } C \subseteq \mathrm{B}(\mathrm{x}, \mathrm{r})\} \text {. }
$$

Theorem 4.3. Let $k>0$ and let $(X, d)$ be a complete $C A T(k)$ space with $\mathrm{rad}(X)<D_{k} / 2$. Then each nonexpansive mapping $T: X \rightarrow 2^{X} \backslash\{\emptyset\}$ with compact values has at least one fixed point.

Proof. Take $x_{0} \in X$ in such a way that $\sup _{y \in X} d\left(x_{0}, y\right)<D_{k} / 2$. As it is shown in [14, Lemma $3]$, it is enough to take $s(t)=\sin (t \pi / 2)$ for $t \in(0,1)$ to verify condition (i) with $x=x_{0}$ in $X$. In a similar manner as above we obtain an approximate fixed point sequence $\left\{x_{n}\right\}$. On account 
of $\operatorname{rad}\left(\left\{x_{n}\right\}\right) \leq \operatorname{rad}(X)$ and [15, Proposition 4.1] the asymptotic center of each subsequence of $\left\{x_{n}\right\}$ is unique, and the rest of the proof is not different from the case of uniformly convex Banach spaces.

Next we consider two further conditions to guarantee the existence of fixed points for nonexpansive set-valued mappings with compact values in uniformly convex metric spaces.

(ii) There is a function $f: \mathbb{N} \times \mathbb{R}_{+} \rightarrow \mathbb{R}_{+}$such that

$$
\lim _{\delta \rightarrow 0^{+}} f(n, \delta)=0, \quad n \in \mathbb{N},
$$

and for all $x, y, z \in X$ and $u \in[x, y], v \in[x, z]$ was chosen in such a way that $d(x, u)=n^{-1} d(x, y), d(x, v)=n^{-1} d(x, z)$, and $d(u, v) \leq \delta$, we have

$$
d(y, z) \leq f(n, \delta)
$$

(iii) $\lim _{r \rightarrow R^{+}, \varepsilon \rightarrow 2^{-}} \delta(r, \varepsilon)=1$, where $\delta(r, \varepsilon)$ is, as usual, a monotone modulus of convexity of the space.

Remark 4.4. Notice that, roughly speaking, conditions (i) and (ii) give opposite information about the geometry of the space. While condition (i) implies that geodesic emanating from a same point must separate and no matters how fast, condition (ii) imposes a superior bound about how much two geodesics emanating from a same point are allowed to separate. It is easy to see that any geodesic space admitting bifurcating geodesics cannot verify condition (ii). In particular, condition (ii) does not hold in $\mathbb{R}$-trees. It easily follows from the definitions that geodesic spaces with curvature bounded below by a real number $k$ (check [16] for a detailed exposition about these spaces) satisfy condition (ii).

Before stating our next result we need to introduce a definition.

Definition 4.5. A geodesic space $X$ is said to have the geodesic extension property if any geodesic segment in $X$ is actually contained in a geodesic line, that is, in a geodesic $\gamma: \mathbb{R} \rightarrow$ $X$.

Theorem 4.6. Let $X$ be a complete uniformly convex metric space with a monotone modulus of convexity and the geodesic extension property. Moreover, suppose that $X$ satisfies conditions (ii) and (iii) and $C$ is a nonempty bounded closed and convex subset of X. If T:C $\rightarrow 2^{C} \backslash\{\emptyset\}$ is a nonexpansive mappings with compact values then $T$ has at least one fixed point.

Proof. From the proof of Theorem 4.2, we know that if $\inf _{x \in C} \operatorname{dist}(x, T(x))=0$ then the conclusion follows. So let us suppose that

$$
d:=\inf _{x \in C} d(x, T(x))>0
$$

and fix $\varepsilon>0$ small enough. Let $x_{0}$ be chosen in such a way that $d\left(x_{0}, T\left(x_{0}\right)\right)<d+\varepsilon$ and let us denote this distance by $d_{1}$. Clearly, from the compactness of $T\left(x_{0}\right)$ it follows the existence of $x_{1} \in T\left(x_{0}\right)$ for which $d\left(x_{0}, x_{1}\right)=d_{1}$. Now assume that $m_{0}$ is a midpoint of the metric 
segment $\left[x_{0}, x_{1}\right]$. The conditions imposed on $T$ imply that there is $m_{1} \in T\left(m_{0}\right)$ such that $d\left(x_{1}, m_{1}\right) \leq d_{1} / 2$ and $d\left(m_{0}, m_{1}\right)=d_{2} \geq d$.

To estimate the distance between $x_{0}$ and $m_{1}$ let us consider the midpoint $p_{0}$ of $\left[m_{0}, m_{1}\right]$. Using the uniformly convexity of $X$ we obtain that

$$
d\left(x_{1}, p_{0}\right) \leq\left(1-\delta\left(\frac{d_{1}}{2}, \frac{2 d}{d_{1}}\right)\right) \frac{d_{1}}{2}=: \delta_{1}
$$

If we denote by $x_{3 / 2}$ the point of the geodesic ray $\gamma$ containing $\left[x_{0}, x_{1}\right]$ such that $d\left(x_{1}, x_{3 / 2}\right)=$ $d_{1} / 2$ and $x_{3 / 2} \neq m_{0}$, then $d\left(m_{1}, x_{3 / 2}\right) \leq f\left(2, \delta_{1}\right)$ and

$$
d\left(x_{0}, m_{1}\right) \geq \frac{3}{2} d-f\left(2, \delta_{1}\right)
$$

Repeating our reasoning for $m_{0}, m_{1}$, and $p_{0}$, we obtain points $p_{1} \in T\left(p_{0}\right)$ and $m_{3 / 2} \in X$ satisfying

$$
d\left(p_{1}, m_{3 / 2}\right) \leq f\left(2, \delta_{2}\right), \quad d\left(m_{0}, m_{3 / 2}\right)=\frac{3}{2} d_{2}
$$

where

$$
\delta_{2}=\left(1-\delta\left(\frac{d_{2}}{2}, \frac{2 d}{d_{2}}\right)\right) \frac{d_{2}}{2}
$$

If we denote by $x_{2}$ the point in $\gamma$ for which $d\left(x_{0}, x_{2}\right)=2 d_{1}$ and $d\left(x_{1}, x_{2}\right)=d_{1}$, then it is easy to see that $d\left(x_{2}, m_{3 / 2}\right) \leq f\left(3, \delta_{1}\right)$. Hence

$$
d\left(x_{0}, p_{1}\right) \geq d\left(x_{0}, x_{2}\right)-d\left(x_{2}, m_{3 / 2}\right)-d\left(m_{3 / 2}, p_{1}\right) \geq 2 d-f\left(3, \delta_{1}\right)-f\left(2, \delta_{2}\right) .
$$

The previous procedure gives us induction sequences of points $\left\{x_{n / 2}\right\}$ and numbers $\left\{\delta_{n}\right\}$ such that

$$
d\left(x_{0}, x_{n / 2}\right)=\frac{n}{2} d_{1}
$$

and for $n$ fixed, a point $y \in C$ for which

$$
d\left(y, x_{n / 2}\right) \leq \sum_{k=2}^{n-1} f\left(k, \delta_{n-k}\right)
$$

Next let us fix $M>0$ and find $N \in \mathbb{N}$ such that $M<(N-2)(d / 2)$. Obviously, according to (ii), one can choose $\delta>0$ for which

$$
\sum_{k=1}^{N} f(k, \delta)<d
$$


and by (iii) select $\varepsilon>0$ such small that $(1-\delta(r, d / r))((d+\varepsilon) / 2)<\delta$ for all $r \in(d / 2$, $(d+\varepsilon) / 2]$.

Choosing $x_{0} \in C$ such that $d\left(x_{0}, T\left(x_{0}\right)\right)<d+\varepsilon$ and repeating our iterative procedure $N$ times, one may notice that the sequence $\left\{d_{n}\right\}_{n=1}^{N}$ decreases so $\delta_{n}<\delta$ for each $n \in\{1, \ldots, N\}$. Finally we find $y \in C$ for which, on account of (4.15),

$$
\begin{gathered}
d\left(y, x_{N / 2}\right) \leq \sum_{n=1}^{N} f(n, \delta) \\
d\left(x_{0}, y\right) \geq N \frac{d}{2}-\sum_{n=1}^{N} f(n, \delta)>(N-2) \frac{d}{2}>M
\end{gathered}
$$

contrary to the boundedness of $C$. Hence $d=0$ and the result follows.

From Theorems 3.11 and 4.2 one may get the following generalization of [1, Theorem 5.2]. We omit the proof as it is analog to the one given for CAT(0) spaces in [1]. We first need some notations and definitions.

Let $X$ be a uniformly convex metric space. Consider the mappings $t: X \rightarrow X$ and $T: X \rightarrow 2^{X} \backslash\{\emptyset\}$, then $t$ and $T$ are said to be commuting mappings if $t(y) \in T(t(x))$ for all $y \in T(x)$ and for all $x \in X$. A point $z$ is called a center for the mapping $t: X \rightarrow X$ if for each $x \in X, d(z, t(x)) \leq d(z, x)$. The set $Z(t)$ denotes the set of all centers of the mappings $t$.

Theorem 4.7. Let $(X, d)$ be a bounded and complete uniformly convex space with a monotone modulus of convexity for which condition (i) holds. Suppose that $t: X \rightarrow X$ is pointwise asymptotically nonexpansive and $T: X \rightarrow 2^{X} \backslash\{\emptyset\}$ a nonexpansive mapping with compact and convex values. If $t$ and $T$ commute and satisfy the condition

$$
T(x) \cap \operatorname{Fix}(t) \subset Z(t), \quad x \in \operatorname{Fix}(t),
$$

then there is $z \in X$ such that $z=t(z) \in T(z)$.

Remark 4.8. The same result remains true if the uniformly convex metric space is supposed to have the geodesic extension property, and conditions (ii)-(iii) hold instead of condition (i).

We finish this work with a last remark about a different condition to obtain another version of Theorem 4.6. Let $X$ be a metric space. Then we say that $X$ has the Stečkin property if for $\varepsilon, d$, and $r$ fixed positive numbers there exist $\xi=\xi(\varepsilon, d, r)>0$ such that if $x, y \in X$ satisfy $d(x, y)=r$, then

$$
\operatorname{diam}[B(y, d+\xi) \backslash B(x, d+r)]<\varepsilon .
$$

The Stečkin property was introduced in [17] to obtain different results regarding the existence of unique nearest and farthest points to closed subsets of normed linear spaces. This property has been studied by many authors since then being of special relevance in the study of uniformly convex Banach spaces; see for instance $[18,19]$. It is known that the Stečkin property does not happen in geodesic spaces with bifurcating geodesics $[19,20]$ and it has 
been shown to be related to the property of having curvature bounded below (see [16] for details about spaces with curvature bounded below); see $[19,20]$. We next show that this property, in addition to a similar condition to (ii), leads to the existence of fixed points for set-valued nonexpansive mappings. We first introduce condition (ii)':

(ii)' there is a function $f: \mathbb{N} \times \mathbb{R}_{+} \rightarrow \mathbb{R}_{+}$such that

$$
\lim _{\delta \rightarrow 0^{+}} f(n, \delta)=0, \quad n \in \mathbb{N},
$$

and for all $x, y, z \in X$ and $u \in[x, y], v \in[x, z]$ was chosen in such a way that $d(x, u)=(2 / n) d(x, y), d(x, v)=(2 / n) d(x, z)$, and $d(u, v) \leq \delta$, we have

$$
d(y, z) \leq f(n, \delta)
$$

Theorem 4.9. Let $X$ be a complete uniformly convex metric space with a monotone modulus of convexity and the geodesic extension property. Moreover, suppose that $X$ satisfies conditions (ii)' and the Steckin property. Let $C$ be a nonempty bounded closed and convex subset of X. If T:C $\rightarrow 2^{C} \backslash\{\emptyset\}$ is a nonexpansive mappings with compact values then $T$ has at least one fixed point.

Proof. We will omit details for this proof as it follows the same patterns of the proof of Theorem 4.6. We will just point out that the main difference happens when the modulus of convexity is used to estimate $d\left(x_{0}, m_{1}\right)$ first (see (4.10)), and $d\left(y, x_{n / 2}\right)$ later (see (4.15)), now we use property $\mathrm{S}$ to achieve similar estimations.

\section{Note Added in Proof}

To prove (3.16) in Proposition 3.13 we need to ask for something more, in particular, it suffices if we assume that $T$ is uniformly continuous. Under this assumption we can prove by induction that $\left\{T^{m}\left(x_{n}\right)\right\}$ is an approximate fixed point sequence for each $m \in \mathbb{N}$. Indeed, let $m=1, \varepsilon>0$ and choose $\delta=\delta(\varepsilon)>0$ the one given by the uniform continuity of $T$. Since $\left\{x_{n}\right\}$ is an approximate fixed point sequence, there exists $n_{0} \in \mathbb{N}$ such that $d\left(x_{n}, T\left(x_{n}\right)\right) \leq \delta$ for every $n \geq n_{0}$. This implies that

$$
d\left(T\left(x_{n}\right), T^{2}\left(x_{n}\right)\right) \leq \varepsilon
$$

for every $n \geq n_{0}$. Thus $\left\{d\left(T\left(x_{n}\right), T^{2}\left(x_{n}\right)\right)\right\} \rightarrow 0$ which proves our claim. Suppose now that $\left\{T^{m-1}\left(x_{n}\right)\right\}$ is an approximate fixed point sequence. We want to see that $\left\{T^{m}\left(x_{n}\right)\right\}$ is so too. Given $\varepsilon>0$ we fix $\delta>0$ as in the case $m=1$. Since $\left\{T^{m-1}\left(x_{n}\right)\right\}$ is an approximate fixed point sequence, there exists $n_{0} \in \mathbb{N}$ such that $d\left(T^{m-1}\left(x_{n}\right), T^{m}\left(x_{n}\right)\right) \leq \delta$ for every $n \geq n_{0}$. This implies that

$$
d\left(T^{m}\left(x_{n}\right), T^{m+1}\left(x_{n}\right)\right) \leq \varepsilon
$$

for every $n \geq n_{0}$. Thus $\left\{T^{m}\left(x_{n}\right)\right\}$ is an approximate fixed point sequence. 
Now, since

$$
d\left(x, T^{m}\left(x_{n}\right)\right) \leq d\left(x, x_{n}\right)+d\left(x_{n}, T^{m}\left(x_{n}\right)\right), \quad d\left(x, x_{n}\right) \leq d\left(x, T^{m}\left(x_{n}\right)\right)+d\left(T^{m}\left(x_{n}\right), x_{n}\right),
$$

by recalling that $d\left(T^{m}\left(x_{n}\right), x_{n}\right) \leq \sum_{k=0}^{k=m-1} d\left(T^{k}\left(x_{n}\right), T^{k+1}\left(x_{n}\right)\right)$, (3.16) follows.

\section{Acknowledgments}

The two first authors were partially supported by the Ministery of Science and Technology of Spain, Grant BFM 2000-0344-CO2-01 and La Junta de Antalucía Project FQM-127. This work was carried out while the third author was visiting the University of Seville. She acknowledges the kind hospitality of the Departamento de Análisis Matemático. This work is dedicated to W. A. Kirk.

\section{References}

[1] N. Hussain and M. A. Khamsi, "On asymptotic pointwise contractions in Metric spaces," Nonlinear Analysis: Theory, Methods \& Applications. In press.

[2] M. R. Bridson and A. Haefliger, Metric Spaces of Non-Positive Curvature, vol. 319 of Grundlehren der Mathematischen Wissenschaften, Springer, Berlin, Germany, 1999.

[3] U. Kohlenbach and L. Leuştean, "Asymptotically nonexpansive mappings in uniformly convex hyperbolic spaces," to appear in Journal of the European Mathematical Society, http:/ / arxiv.org/abs/0707.1626.

[4] W. A. Kirk, "Some recent results in metric fixed point theory," Journal of Fixed Point Theory and Applications, vol. 2, no. 2, pp. 195-207, 2007.

[5] M. Arav, F. E. Castillo Santos, S. Reich, and A. J. Zaslavski, “A note on asymptotic contractions," Fixed Point Theory and Applications, vol. 2007, Article ID 39465, 6 pages, 2007.

[6] W. A. Kirk, "Fixed points of asymptotic contractions," Journal of Mathematical Analysis and Applications, vol. 277, no. 2, pp. 645-650, 2003.

[7] M. A. Khamsi, "On asymptotically nonexpansive mappings in hyperconvex metric spaces," Proceedings of the American Mathematical Society, vol. 132, no. 2, pp. 365-373, 2004.

[8] W. A. Kirk and H.-K. Xu, "Asymptotic pointwise contractions," Nonlinear Analysis: Theory, Methods $\mathcal{E}$ Applications, vol. 69, no. 12, pp. 4706-4712, 2008.

[9] T. Shimizu and W. Takahashi, "Fixed point theorems in certain convex metric spaces," Mathematica Japonica, vol. 37, no. 5, pp. 855-859, 1992.

[10] L. Leuştean, "A quadratic rate of asymptotic regularity for CAT(0)-spaces," Journal of Mathematical Analysis and Applications, vol. 325, no. 1, pp. 386-399, 2007.

[11] M. A. Khamsi and W. A. Kirk, An Introduction to Metric Spaces and Fixed Point Theory, Pure and Applied Mathematics, Wiley-Interscience, New York, NY, USA, 2001.

[12] L. Leuştean, "Nonexpansive iterations in uniformly convex W-hyperbolic spaces," to appear in Contemporary Mathematics, http://arxiv.org/abs/0810.4117.

[13] K. Goebel and W. A. Kirk, Topics in Metric Fixed Point Theory, vol. 28 of Cambridge Studies in Advanced Mathematics, Cambridge University Press, Cambridge, UK, 1990.

[14] B. Piątek, "Halpern iteration in spherical spaces," submitted to publication.

[15] R. Espínola and A. Fernández-León, "CAT(k)-spaces, weak convergence and fixed points," Journal of Mathematical Analysis and Applications, vol. 353, no. 1, pp. 410-427, 2009.

[16] D. Burago, Y. Burago, and S. Ivanov, A Course in Metric Geometry, vol. 33 of Graduate Studies in Mathematics, American Mathematical Society, Providence, RI, USA, 2001.

[17] S. B. Stečkin, "Approximation properties of sets in normed linear spaces," Revue Roumaine de Mathématiques Pures et Appliquées, vol. 8, pp. 5-18, 1963 (Russian).

[18] F. S. de Blasi, J. Myjak, and P. L. Papini, "Porous sets in best approximation theory," Journal of the London Mathematical Society, vol. 44, no. 1, pp. 135-142, 1991. 
[19] A. Kaewcharoen and W. A. Kirk, "Proximinality in geodesic spaces," Abstract and Applied Analysis, vol. 2006, Article ID 43591, 10 pages, 2006.

[20] T. Zamfirescu, "On the cut locus in Alexandrov spaces and applications to convex surfaces," Pacific Journal of Mathematics, vol. 217, no. 2, pp. 375-386, 2004. 